This is a postprint version of the following published document:

Kobayashi, H., et al. (eds.) (2014). Proceedings of the 8th International Symposium on Impact Engineering. (pp.310-315). (Applied Mechanics and Materials, 566). Switzerland: Trans Tech Publications.

DOI http://dx.doi.org/10.4028/www.scientific.net/AMM.566.310

(C) 2014 Trans Tech Publications 


\title{
Numerical analysis of the brittle-ductile transition in the failure-mode in polymeric materials.
}

\author{
Josué Aranda-Ruiz ${ }^{1, a}$ and José A. Loya ${ }^{1, b}$ \\ ${ }^{1}$ Department of Continuum Mechanics and Structural Analysis, University Carlos III of Madrid. \\ Avda. de la Universidad 30, 28911 Leganés, Madrid, Spain \\ ajaranda@ing.uc3m.es, bjloya@ing.uc3m.es
}

Keywords: Polymers; Damage Models; Failure-Mode Transition.

\begin{abstract}
In this paper we analyze, using the Finite Element Method, the process of brittle-ductile transition in the failure mode observed in polycarbonate notched specimens under impact loads. In order to analyze this transition we have implemented, through a user subroutine, a damage model which combines a tensional fracture criterion and an energetic, acting simultaneously. The competition between both criteria predicts the difference in material behavior from a critical impact velocity, and how this transition is produced on different planes through the thickness of the specimen. These results show the necessity of employing three-dimensional models for its study.
\end{abstract}

\section{Introduction}

The good relationship between the impact resistance of certain polymeric materials with respect to their weight and cost has prompted its use in the industry over the last decades. During its service lifetime, these components can be subjected to dynamic and impact loads, which requires a deeper understanding of the mechanical behavior of these materials under high strain rates, as well as their fracture behavior. Polymeric materials have called the attention from the scientific community $[1,2,3,4,5]$, showing the importance to predict the failure conditions of a component.

The brittle-ductile transition in the failure mode has been studied mainly in metals [6,7], but it has been also observed in polymeric materials, such as PolyMethylMethAcrylate (PMMA) [8] and PolyCarbonate (PC) [9]. This phenomenon of transition in the failure mode was analyzed by Kalthoff [6] in steel specimens subjected to dynamic mode-II loading. He observed that at low impact velocities, the fracture mechanism of cleavage was the dominant, and cracks propagated at an angle of about 70 degrees relative to the plane of the notch. However, from a certain impact velocity, adiabatic shear bands appear and cracks propagate at a much smaller angle, about $10^{\circ}$. The change in the failure mode, from a mechanism of brittle fracture to a ductile one, is called brittleductile transition in the failure mode.

Most published analysis $[6,8,9]$, either on metals or polymers, focus on the qualitative description of the phenomenon. Recently, Dolinski et al. [7] proposed a damage model applied to metallic materials able to predict this transition. However, as it has been experimentally observed, the failure modes produced in polymeric materials are similar to those reported by [7], so it might be interesting to extend the proposed methodology to polymers.

In this paper, using the Finite Element Method, a full 3D numerical model has been build, to reproduce tests made on PC simple notched specimens [9], incorporating a damage model by means of a user subroutine. This damage model is an extension of the one published by Dolinski et al. [7], to be applicable to polymeric materials, in particular the PC. In the analysis, a special attention to the process of brittle-ductile transition in the failure mode has been paid. The results highlight the distinctly three-dimensional character of the crack propagation processes and the need of use 3D models for its study. 


\section{Description of the problem}

The numerical simulations carried out in this work correspond to the experimental tests performed by Ravi-Chandar et al. [9]. Such tests involve the asymmetric impact of a cylindrical projectile (length $100 \mathrm{~mm}$, diameter $50 \mathrm{~mm})$ against a flat and simple notched specimen $(100 \times 400 \times 6 \mathrm{~mm})$, both of PC, as shown in Fig. 1. The impact occurs in the upper half of the specimen, considering theoretically null the impact height relative to the plane of the notch, and imposing no vertical displacement on the bottom surface of the specimen.

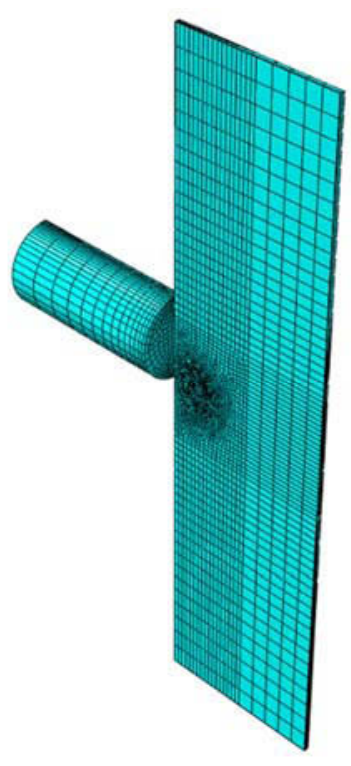

Fig.1 Three-dimensional model of the problem

\section{Damage model}

The implemented damage model is an adaptation from the one published by Dolinski et al. [7]. The model incorporates simultaneously two modes of failure, on the one hand, a brittle fracture criterion defined from the maximum principal stress, and secondly, a ductile fracture criterion (adiabatic shear band), defined from the density strain energy.

Brittle fracture criterion. It is defined using a local failure criterion based on the maximum principal stress, so that when the maximum principal stress, at some point of the solid, reaches a critical value, $\sigma_{I}^{\text {crit }}$, the failure of the element occurs, as stated in Eq. 1.

$$
\sigma_{I}=\sigma_{I}^{c r i t}(\dot{\bar{\varepsilon}}) \text {. }
$$

The last expression considers the dependence of $\sigma_{I}^{\text {crit }}$ with the equivalent strain rate $\dot{\bar{\varepsilon}}$, so it is necessary to know the stress-strain state in an area that surrounds the notch tip [10]. The relationship between $\sigma_{I}^{\text {crit }}$ and $\dot{\bar{\varepsilon}}$ can be obtained from experimental results [9].

Ductile fracture criterion. The ductile fracture criterion is based on the density of strain energy, and sets the gradual deterioration of the structural strength of an element in accordance with the following expression:

$$
\tilde{\sigma}_{e q}=\tilde{\sigma}_{e q}^{*}(1-D) \text {, }
$$

where $\tilde{\sigma}_{e q}^{*}$ is the equivalent stress at the present moment, for a given equivalent strain, $\tilde{\varepsilon}_{e q} \geq \tilde{\varepsilon}_{e q}^{\text {crit }}$ (being $\tilde{\varepsilon}_{e q}^{\text {crit }}$ the equivalent strain from which the structural strength begins to deteriorate). The parameter $D \in[0,1]$ denotes the level of damage present in the element, implying that for $D=0$ the element is intact, while if $D=1$ the element have lost all its resistive capacity. This parameter $D$ is given by the following equation: 


$$
D=\frac{W-W_{\text {crit }}}{W_{\text {frac }}-W_{\text {crit }}}
$$

where $W_{\text {frac }}$ corresponds to the energy density when the entire rupture of the element occurs, that is, when the equivalent stress reaches a value equal to zero, and $W_{\text {crit }}$ determines a critical level of the total density of strain energy stored in an element, given by the following expression:

$$
W_{c r i t}=\int_{0}^{\tilde{\varepsilon}_{e q}^{c r i t}} \sigma_{i j} d \varepsilon_{i j}
$$

being $\sigma_{i j}$ and $\varepsilon_{i j}$ the components of the stress and strain tensors respectively. The different values for energy densities are obtained in order to reproduce experimental results published [9].

\section{Numerical model}

In this work, a full three-dimensional numerical model (specimen and projectile) of the problem analyzed by Ravi-Chandar et al. [9], has been implemented in the finite element commercial code ABAQUS/Explicit v6.11. Due to the symmetry of the problem, only half of the specimen and the projectile have been considered, imposing the corresponding boundary conditions. In order to consider the high stress and strain gradients that occur near the end of the notch, the mesh has been refined in this area. In total, the mesh of the specimen consists of 183678 nodes and 165030 elements (C3D8RT) while the projectile consists of 3990 nodes and 3220 elements (C3D8R).

Constitutive behavior of the PC. To reduce the computational cost of numerical simulations, the PC has been modeled as linear elastic for the projectile, and as thermoviscoplastic material, defined by the stress-strain law contained in the Eqs. 5-9, for the specimen.

$$
\begin{aligned}
& \sigma=\left\{\begin{array}{llr}
\sigma_{1} & \text { if } & \begin{array}{r}
\varepsilon \leq \frac{\sigma_{y}}{E} \\
\sigma_{2}
\end{array} \text { if } \\
\sigma_{3} & \text { if } & 0.81 \varepsilon_{h}+\frac{\sigma_{y}}{E}-\varepsilon_{0} \leq \varepsilon \leq \varepsilon_{h}+\frac{\sigma_{y}}{E}-\varepsilon_{0} \\
\sigma_{4} & \text { if } & \varepsilon \geq \varepsilon_{h}+\frac{\sigma_{y}}{E}-\varepsilon_{0}
\end{array}\right. \\
& \sigma_{1}=E \varepsilon, \\
& \sigma_{2}=\sigma_{y}+A\left(\varepsilon-\frac{\sigma_{y}}{E}\right), \\
& \sigma_{3}=\sigma_{y}+A\left(0.81 \varepsilon_{h}-\varepsilon_{0}\right)+B\left(\varepsilon-0.81 \varepsilon_{h}-\frac{\sigma_{y}}{E}+\varepsilon_{0}\right), \\
& \sigma_{4}=\sigma_{y}+A\left(0.81 \varepsilon_{h}-\varepsilon_{0}\right)+0.19 B \varepsilon_{h}+E\left(\varepsilon-\varepsilon_{h}-\frac{\sigma_{y}}{E}+\varepsilon_{0}\right) .
\end{aligned}
$$

The temperature [9] and the strain rate [11] dependences are given by the following expressions:

$$
\begin{aligned}
& \sigma_{y}(\theta)=E \varepsilon_{0}-\beta\left(\theta-\theta_{0}\right), \\
& \sigma_{y}(\dot{\varepsilon})=E \varepsilon_{0}+\frac{2 k \Theta}{V} \sinh ^{-1}\left(\frac{\dot{\varepsilon}}{\dot{\varepsilon}^{*}}\right)^{1 / n},
\end{aligned}
$$

being $\theta_{0}=20^{\circ} C$ the reference temperature. Table 1 shows the mechanical properties used for PC. 
Table 1 Parameters of constitutive behavior of PC

\begin{tabular}{|c|c|c|c|c|c|}
\hline Tensile modulus & $E$ & $2.4[\mathrm{GPa}]$ & Yield strain & $\varepsilon_{0}$ & 0.021 \\
\hline Density & $\rho$ & $1160\left[\mathrm{~kg} / \mathrm{m}^{3}\right]$ & Strain at molecular orientation & $\varepsilon_{h}$ & 0.521 \\
\hline Poisson's ratio & $v$ & 0.34 & Coefficient of thermal softening & $\beta$ & $0.25[\mathrm{MPa} / \mathrm{K}]$ \\
\hline Constitutive law constant & $A$ & $170\left[\mathrm{MJ} / \mathrm{m}^{3}\right]$ & Specific Heat & $C_{p}$ & $1170[\mathrm{~J} /(\mathrm{kg} \cdot \mathrm{K})]$ \\
\hline Constitutive law constant & $B$ & $100[\mathrm{MPa}]$ & Thermal conductivity & $k$ & $0.2[\mathrm{~W} /(\mathrm{K} \cdot \mathrm{m})]$ \\
\hline Yield stress in tension & $\sigma_{y}^{t}$ & $50.4[\mathrm{MPa}]$ & Coefficient of thermal expansion & $\alpha$ & $6.5 \cdot 10^{-5}\left[\mathrm{~K}^{-1}\right]$ \\
\hline Yield stress in compression & $\sigma_{y}^{c}$ & $57[\mathrm{MPa}]$ & Coefficient of Quinney-Taylor & $\varphi$ & 0.6 \\
\hline
\end{tabular}

\section{Results}

Once the model proposed is calibrated from the experimental results obtained by Ravi-Chandar et al. [9], the process of brittle-ductile transition in the failure mode has been analyzed in detail.

Brittle fracture mode. Fig. 2 shows the results of an impact at a velocity of $40 \mathrm{~m} / \mathrm{s}$, below the transition velocity, where a brittle fracture by mode-I appears. Both numerical and experimental results exhibit two crack propagation paths, indicated in this figure by the letters B and L. Crack B appears in first place by the impact of the projectile, before the phenomenon of wave reflection from the edges of the specimen occurs. In the numerical and experimental results, this first crack propagates in an angle with the horizontal of about $70^{\circ}$, coinciding with the largest maximum principal stress. Fissure L appears later due to interaction of the waves reflected by the end of the initial notch, propagating in opposite direction to crack B.

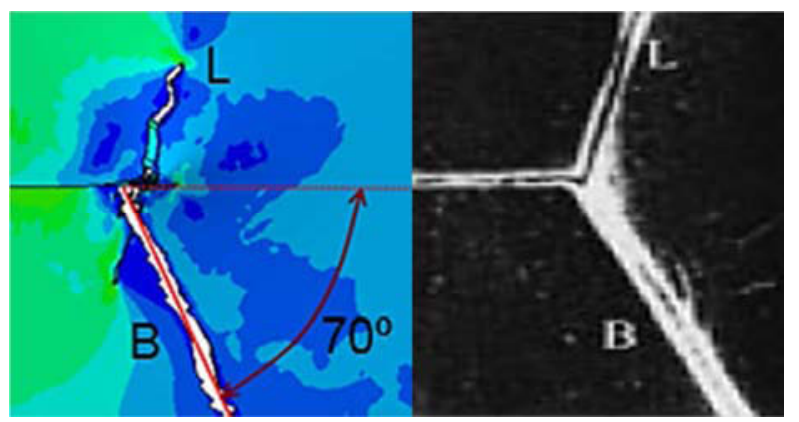

Fig.2 Brittle fracture mode. Numerical (left) and experimental (right) results [9].

Ductile fracture mode. For an impact velocity of $60 \mathrm{~m} / \mathrm{s}$ (above the transition velocity), a ductile fracture mode dominated by adiabatic shear bands appears. Both experimental and numerical results show as the crack propagates in the direction in which maximum plastic deformations and higher temperature rise occur (with the horizontal of about $10^{\circ}$ ), till stop after about some millimeters (Fig. 3).
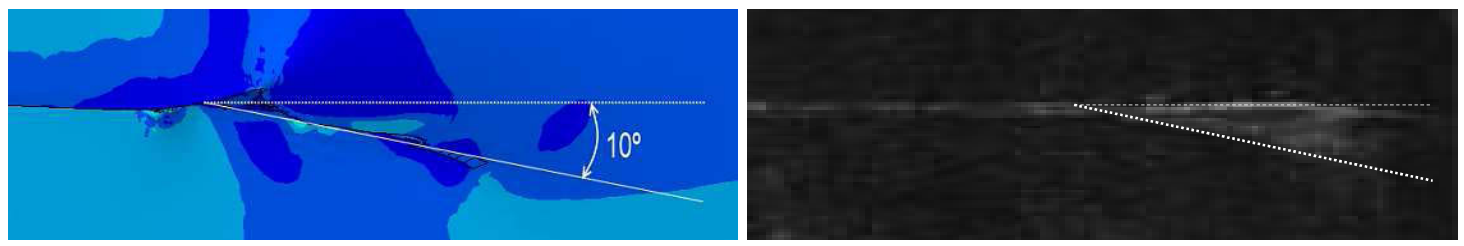

Fig.3 Ductile fracture mode. Numerical (left) and experimental (right) results[9]. 
Process of brittle-ductile transition. Experimentally, the transition velocity for the material and specimen geometry used was determined as $58 \mathrm{~m} / \mathrm{s}$ [9], while in the numerical simulations this is around $55 \mathrm{~m} / \mathrm{s}$, being the estimated error of $5.17 \%$.

Below $55 \mathrm{~m} / \mathrm{s}$, the failure mode is brittle, but as the impact velocity approaches the transition velocity, certain variations can be observed in the process of crack propagation, differing from the free surface of the specimen and its plane of symmetry: a ductile crack appears on the exterior face of the specimen, while in the plane of symmetry appears a crack dominated by brittle failure mode (situation 1). Thereupon, the brittle failure mode is propagated along the thickness of the sample to the outside of it. When the impact velocity increases slightly (situation 2) without exceeding the transition velocity, it can be observed that in both, the free surface and the plane of symmetry, the failure produced is ductile. From a certain moment, it is produced in the plane of symmetry a new transition to a brittle failure mode, which again propagates towards the free surfaces along the thickness of the specimen. These two transitions in the failure-mode have been also experimentally observed by Zhou et al. [3] on Ti-6Al-4V specimens. Therefore it has been observed that the crack which is initiated in the plane of symmetry in situations 1 and 2, propagates along the specimen thickness, an effect that could be observed only by a 3D model, highlighting the three-dimensional character of the crack propagation processes.
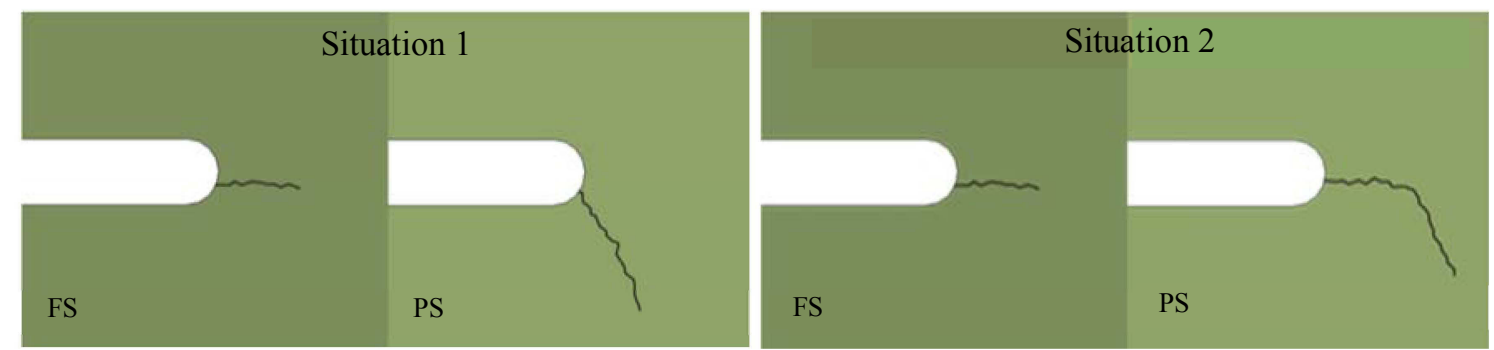

Fig.4 Different situations of crack propagation according to impact velocity.

FS: Free surface. PS: Plane of symmetry.

\section{Conclusions}

In this paper, using the Finite Element Method, a full three-dimensional numerical model of the impact of a cylindrical projectile against PC notched specimens has been implemented, including the damage model, by a user subroutine. It has been proposed a damage model that takes into account simultaneously a local criterion based on stresses and another energetic, which shows the competition between the two modes of failure observed in polymeric materials, and is capable of reproduce the phenomenon of brittle-ductile transition at the failure mode. This damage model is based on another initially applied to metals, and has been adapted to the PC including the difference between tension and compression behavior. Thus, it has been able to reproduce qualitatively, experimental results published in the literature such as: the impact velocity at which the transition in the failure mode occurs, and the direction of crack propagation.

Furthermore, a detailed numerical analysis of the transition between brittle and ductile failure modes has been realized, showing a different behavior in the free sides of the specimen relative to its plane of symmetry. Thus, it has been found as the failure mode occurring in the plane of symmetry propagates along the thickness of the specimen toward the free surface. So, the threedimensional character of the crack propagation processes is highlighted.

\section{Acknowledgements}

Authors thank the Comisión Interministerial de Ciencia y Tecnología of the Spanish Government for the support of this work through the Research Project DPI2011-23191. 


\section{References}

[1] Z. Li and J. Lambros: International Journal of Solids and Structures. Vol. 38 (2001), p. 3549.

[2] J.A. Loya and K. Ravi-Chandar: Anales de Mecánica de la Fractura. Vol. 23 (2006), p. 195.

[3] M. Zhou, A.J. Rosakis and G. Ravichandran: Int. Journal of Plasticity. Vol. 14 (1998), p. 435.

[4] J.A. Loya, E. Villa and J. Fernández-Sáez: Polymer Testing. Vol. 29 (2010), p. 113.

[5] K. Ravi-Chandar and W.G. Knauss: International Journal of Fracture. Vol. 25 (1984), p. 247.

[6] J. Kalthoff: Optical Engineering. Vol. 27 (1988), p. 835.

[7] M. Dolinski, D. Rittel and A. Dorogoy: Journal of Mechanics and Physics of Solids. Vol. 58 (2010), p. 1759.

[8] D. Rittel and A. Brill: Journal of Mechanics and Physics of Solids. Vol. 56 (2008), p. 1401.

[9] K. Ravi-Chandar, J. Lu, B. Yang and Z. Zhu: Int. Journal of Fracture. Vol. 101 (2000), p. 33.

[10] R. Ritchie, J. Knott and J. Rice: Journal of Mechanics and Physics of Solids. Vol. 21 (1973), p. 395.

[11] S. Fu, Y. Wang and Y. Wang: Polymer Testing. Vol. 28 (2009), p. 724. 